Original paper

\title{
Triple channel analysis of Gafchromic EBT3 irradiated with clinical carbon-ion beams
}

\author{
Kim Marina Holm ${ }^{\text {a,b,c,d,*, Eduardo G. Yukihara }}{ }^{\mathrm{e}}$, Md Foiez Ahmed ${ }^{\mathrm{f}}$, Steffen Greilich ${ }^{\mathrm{b}, \mathrm{c}}$, \\ Oliver Jäkel ${ }^{\mathrm{b}, \mathrm{c}, \mathrm{g}}$ \\ ${ }^{a}$ Department of Dosimetry for Radiation Therapy and Diagnostic Radiology, Physikalisch-Technische Bundesanstalt (PTB), Bundesallee 100, Braunschweig D-38116, \\ Germany \\ b Department of Medical Physics in Radiation Oncology, German Cancer Research Center (DKFZ), Im Neuenheimer Feld 280, Heidelberg D-69120, Germany \\ ${ }^{\mathrm{c}}$ Heidelberg Institute for Radiation Oncology (HIRO), Heidelberg, Germany \\ ${ }^{\mathrm{d}}$ Department of Physics and Astronomy, Heidelberg University, Im Neuenheimer Feld 226, Heidelberg D-69120, Germany \\ ${ }^{\mathrm{e}}$ Department of Radiation Safety and Security, Paul Scherrer Institute, Forschungsstrasse 111, Villigen PSI 5232, Switzerland \\ ${ }^{\mathrm{f}}$ Sun Nuclear Corporation, 3275 Suntree Blvd, Melbourne, Florida 32940, USA \\ ${ }^{\mathrm{g}}$ Heidelberg Ion Beam Therapy Center (HIT), University Hospital Heidelberg, Im Neuenheimer Feld 450, Heidelberg D-69120, Germany
}

\section{A R T I C L E I N F O}

\section{Keywords:}

Triple channel analysis

Gafchromic EBT3

Carbon-ion beams

\begin{abstract}
A B S T R A C T
Self-developing radiochromic film is widely used in radiotherapy QA procedures. To compensate for typical film inhomogeneities, the triple channel analysis method is commonly used for photon-irradiated film. We investigated the applicability of this method for Gafchromic ${ }^{\mathrm{TM}}$ EBT3 (Ashland) film irradiated with a clinically used carbon-ion beam. Calibration curves were taken from EBT3 film specimens irradiated with monoenergetic carbon-ion beams of different doses. Measurements of the lateral field shape and homogeneity were performed in the middle of a passively modulated spread-out Bragg peak and compared to simultaneous characterization by means of a 2D ionization chamber array. Additional measurements to investigate the applicability of EBT3 for quality assurance (QA) measurement in carbon-ion beams were performed. The triple-channel analysis reduced the relative standard deviation of the doses in a uniform carbon ion field by $30 \%$ (from $1.9 \%$ to $1.3 \%$ ) and reduced the maximum deviation by almost a factor of 3 (from $28.6 \%$ to $9.8 \%$ ), demonstrating the elimination of film artifacts. The corrected film signal showed considerably improved image quality and quantitative agreement with the ionization chamber data, thus providing a clear rationale for the usage of the triple channel analysis in carbon-beam QA.
\end{abstract}

\section{Introduction}

To ensure that the irradiation parameters in scanned ion beam therapy remain constant, measurements of the shape of dose distributions and their homogeneity in the target region are essential elements of routine quality assurance (QA). ${ }^{1}$ To this end, three-dimensional dosimetry is needed. This can be done by measuring the twodimensional dose distribution at different depths. Due to its high spatial resolution, film is the material of choice, particularly for very small fields [1]. Self-developing radiochromatic film is widely employed in clinical routine [2]. It contains a dye in an active layer that becomes polymerized when exposed to radiation; here, the light absorption properties in the irradiated film region change. The film segments can be read out by a flatbed scanner; no developing process is needed [1]. The most commonly used radiochromatic film is Gafchromic ${ }^{\circledR}$ EBT, as well as its descendants, EBT2 and EBT3 [3].

Film measurements may be subject to the presence of artifacts and material inhomogeneities $[4,5]$. Slight variations in the active layer thickness may add variation to the film signal and hence to the measured dose. One well-established method for reducing the influence of these artifacts on EBT film is the triple channel analysis method, wherein information from all three color channels is used to separate the doseindependent part from the resulting dose distribution. Different approaches to this multichannel method were introduced by Micke et al. [6] and Mayer et al. [7] (both used EBT2), generating identical resulting dose distributions. Other correction methods for channel-independent

\footnotetext{
* Corresponding author.

E-mail address: kim.holm@ptb.de (K.M. Holm).

1 QA: quality assurance
} 
perturbations such as the weighted mean model and the truncated normal distribution model were presented by Méndez et al. [8,9] using both EBT2 and EBT3. The application of these correction methods has been investigated for photon radiotherapy [10-12] as well as for brachytherapy [13], whereas it has not been applied to date for carbonion irradiated film.

Radiotherapy by means of carbon-ion beams and pencil beam scanning allows doses to be applied precisely, thus making irradiation of very fine structures possible [14]. However, unwanted inhomogeneities in the irradiation field are more pronounced for scanned carbon-ion beams than for IMRT or for passively delivered protons, as a smaller spot size is used for carbon ions and since carbon ions show considerably reduced lateral scattering compared to photons or protons. For this reason, it is especially important that a 2D-dosimetry with a high spatial resolution is implemented for scanned carbon-ion beams.

Studies on the dosimetric properties of EBT3 in carbon-ion beams showed a limitation to the application of radiochromatic film in carbonion beams due to a signal dependency on the ionization density [15]. For monoenergetic beams, the response of EBT to carbon ions was about $30 \%$ lower than its response to photons [16]. A quenching effect appears that increases with depth [17]. It was shown that the relative efficiency was dependent on the ion type and its energy, which means that the optical density is uniquely connected to the dose only for some very specific situations. Generally, due to the nuclear fragmentation process in a carbon-ion beam, the relation between the optical density and the dose is more complex and needs additional input data. However, it is possible to use EBT film in carbon-ion beams if restricted to relative dose measurements of lateral distributions with similar ionization densities.

The objective of this work is to demonstrate that the triple channel analysis method described by Micke et al. [6] is essential for the dosimetry of carbon ion beams. If the fine dose structures that are created by the combination of pencil-beam delivery and narrow lateral dose distributions of the pencil beams are to be captured by the film, possibly indicating a problem with the beam delivery, then the artifacts caused by film inhomogeneity need to be eliminated using the triplechannel analysis. Although fields as uniform as in photon beams cannot be achieved with scanning carbon-ion beams, the results will show that the average standard deviation and the maximum deviations for uniform carbon-ion beams are reduced using the triple channel analysis.

\section{Material and methods}

\subsection{Gafchromic EBT3}

In this study, we used Gafchromic EBT3 film (lot number 10031801). EBT3 film is a further development of EBT2 film; while its performance is comparable to EBT2 film, it has a reduced orientation dependence [18]. EBT3 film is nearly equivalent to tissue [19] and has a high maximum spatial resolution of $25 \mu \mathrm{m}$. The resulting combined spatial resolution of the film and the readout system may be lower, depending on the performance of the readout system. EBT3 film consists of an active $28 \mu \mathrm{m}$ layer sandwiched between two $125 \mu \mathrm{m}$ matte-polyester layers [20].

\subsection{Irradiation}

All film specimens were irradiated at the Heidelberg Ion Beam Therapy Center $(\mathrm{HIT})^{2}$ [21] using pencil beam scanning [14]. Eight fluences (number of primary particles) in the range of $(0.05-1.5) \cdot 10^{6}$ $\mathrm{mm}^{-2}$ corresponding to absorbed doses to water between 0.1 and $3.2 \mathrm{~Gy}$ (measured with a calibrated ionization chamber) at a depth of $2.9 \mathrm{~mm}$ water-equivalent thickness were used to establish the calibration. The beam had an initial energy of $278.29 \mathrm{MeV} / \mathrm{u}$. In addition, film specimens were also irradiated using a 6 MV LINAC (Elekta Synergy), a calibration field size of $10 \times 10 \mathrm{~cm}^{2}$ and doses in the range of 0.5-3.0 Gy (determined from the LINAC's monitor units) at a $10 \mathrm{~cm}$ depth in a waterequivalent slab phantom (RW-3, PTW, Germany).

The carbon-ion irradiation field of interest was optimized for homogeneity, as it was also applied for the study presented in [22]. It consisted of four monoenergetic layers of $36 \times 36$ spots in a rectangular grid with $2 \mathrm{~mm}$ spacing, which were shifted against each other by $1 \mathrm{~mm}$ in the $\mathrm{x}$-, $\mathrm{y}$ - and xy-directions. The beam's initial energy was 278.29 $\mathrm{MeV} / \mathrm{u}$. A spread-out Bragg peak (SOBP) ${ }^{3}$ was generated by a range modulator consisting of pyramid-shaped pins [23]. In total, a dose of 1.5 Gy was applied to a field size of $6 \times 6 \times 6 \mathrm{~cm}^{3}$.

In addition to the homogeneous field, EBT3 measurements were performed for a ${ }^{12} \mathrm{C}$ patient treatment plan. ${ }^{12} \mathrm{C}$ patient treatment plans delivered by means of active beam scanning as used at HIT consist of several iso-energy slices. This results in a spectrum of the traversing ions' residual ranges and hence the ionization density at one depth. As EBT3 shows a quenching of the signal depending on the ionization density $[17,15]$, the relative dose within one slice in depth would be over-/underestimated. To avoid this effect, we irradiated only one single iso-energy slice per film measurement for a somewhat constant ionization density at the measurement depth. Here, the whole patient treatment plan consists of 21 iso-energy slices with initial beam energies between $166.1 \mathrm{MeV} / \mathrm{u}$ and $248.9 \mathrm{MeV} / \mathrm{u}$, the iso-energy slice used had an initial beam energy of $212.12 \mathrm{MeV} / \mathrm{u}$; a maximum dose of $1.25 \mathrm{~Gy}$ was applied. EBT3 specimens were positioned between solid water (RW3, PTW, Germany) slabs corresponding to $75 \mathrm{~mm}$ water-equivalent depth. An extra calibration curve at this depth was estimated following the same procedure as described above. Care was taken to position all film specimens in the same orientation relative to the beam.

\subsection{Scanning}

The film specimens were scanned on a flatbed Epson Expression $10000 \mathrm{XL}$ scanner in transmission mode after at least $32 \mathrm{~h}$ postirradiation, as a post-irradiation of at least $24 \mathrm{~h}$ is recommended (preferably $48 \mathrm{~h}$ ) [24]. Before each scanning procedure, 10 pre-scans of the whole scanning field were performed to scan all the film specimens at a constant working temperature $[25,26]$.

Each film specimen was scanned separately and positioned in the center of the scanner to reduce lateral scan artifacts [27]. To keep the specimen flat on the scanner, a glass compression plate was positioned on top of the specimen [28]. The specimens were scanned with a resolution of $72 \mathrm{dpi}$ and a 48-bit color depth. A central ROI of 56 x $56 \mathrm{px}$ for the calibration fields was selected. All color-correction features were deactivated and the specimens were scanned as positive film in transparency mode as recommended by the Gafchromic Film QA Protocol [29] as well as by Micke et al. [6]. Each specimen was scanned once, since the reproducibility of scanning investigated in a pre-study was found to be $0.1 \%$ of the maximum.

From the scanned pixel values $P$, the optical density $O D$ for each pixel was calculated using

$O D=\log _{10} \frac{P_{\text {blank }}}{P}$

with $P_{\text {blank }}=65325$ taken from a flood field image (i.e., nothing was placed on the scanner). From that, the net optical density netOD could be calculated, considering the zero dose signal of the unexposed film:

$n e t O D=O D_{\text {exp }}-O D_{\text {unexp }}$,

with $O D_{\exp }$ and $O D_{\text {unexp }}$ the optical densities of an exposed and an un- 
exposed film specimen. As the information about film thickness and homogeneity is included in the zero dose signal, the optical density has to be used for the triple channel analysis instead of the netOD.

\subsection{Calibration}

For each calibration field, the mean value of the optical density determined was calculated for the central ROI selected (see Section 2.3). The dose delivered for each calibration field was plotted as a function of this mean value. To obtain the dose from the optical density, Eq. (3) was fitted to this plot:

$D(O D)=\frac{c \cdot 10^{-O D}-a}{b-10^{-O D}}$,

yielding the fitting parameters $a, b$ and $c$ for each color channel as well as their covariance matrix, from which their standard deviations were derived. The resulting calibration curves can be seen in Fig. 1.

\subsection{Triple channel analysis}

A disturbance value $\delta$ was introduced as follows:

$D_{X}=f_{X}\left(O D_{X} \cdot \delta\right)$,

with $D_{X}$ and $O D_{X}$ denoting the dose and optical density of the color channel $X$ and $f_{X}$ denoting the corresponding calibration function as given by Eq. (3). $\delta$ describes the dose-independent disturbance in the measured optical density caused by film or scanning artifacts. Multiplying $\delta$ with the optical density removes this disturbance from the dosedependent part of the signal. For a reliable result, the calibration condition for the average disturbance $\bar{\delta}=1$ has to be fulfilled, as is the case for a measurement area sufficiently large to reflect average response of the system [6]. $\delta$ was determined by minimizing the differences in the doses calculated for the three color channels (red, green and blue). To this end, Eq. (5) was solved:

$\Omega(\delta)=\min \left(\sum_{i \neq j}\left(D_{X_{i}}-D_{X_{j}}\right)^{2}\right)$,

with $X_{i}, X_{j} \in\{R, G, B\}$ denoting the three color channels (red, green and blue) [6]. $D_{X_{i}}$ and $D_{X_{j}}$ are given by Eq. (4). For the minimization, the constraint $0.8 \leqslant \delta \leqslant 1.2$ was set to avoid diverging calculations due to artifacts in the scans [30]. As the main absorption band was at $633 \mathrm{~nm}$ [31], the optical density obtained from the film was greatest for the red color channel [1]. Therefore, the corrected film dose distribution was calculated according to Eq. (4) for the red color channel multiplying the optical density from the red channel by the value obtained for $\delta$.

\subsection{IC array}

Measurements were performed simultaneously using a prototype PTW Octavius 1000 P ionization chamber (IC) ${ }^{4}$ array with the film specimen positioned directly on the array. Due to the ICs' effective measuring point at $5.5 \mathrm{~mm}$ water-equivalent depth beneath the array's surface, the measurement depth of the film specimen differs slightly from the IC array's.

In contrast to the commercially available Octavius 1000SRS, the $1000 \mathrm{P}$ was adapted for the application in particle beams; the chambers were filled with air instead of a liquid, thus avoiding recombination effects [32]. The Octavius 1000P consists of 977 ICs arranged in a $10 \mathrm{x}$ $10 \mathrm{~cm}^{2}$ rectangle operated at $1000 \mathrm{~V}$. The ICs are separated by a distance of $2.5 \mathrm{~mm}$ in the inner $5 \times 5 \mathrm{~cm}^{2}$ and $5 \mathrm{~mm}$ in the outer part of the detecting area [33]. Each IC has an active area of $2.3 \times 2.3 \mathrm{~mm}^{2}$, an air-

\footnotetext{
${ }^{4}$ IC: ionization chamber
}

filled gap of $1.8 \mathrm{~mm}$ thickness acts as the active medium [34].

To compare the resulting film signal with the dose distribution that had been measured using the IC array, we performed a gamma index analysis for each measurement in accordance with [35]. A criterion of $3 \%(3 \mathrm{~mm})$ was applied.

\subsection{Monte Carlo FLUKA transport code}

Monte Carlo (MC) ${ }^{5}$ simulations were performed using the FLUKA code version 2011.2x.5 [36]. Single energy slices taken from the patient treatment plan were simulated to compare the resulting dose distribution with the measured film signal. For all simulations, the settings for precise simulations (PRECISIO) were used. The original irradiation plan was implemented using a FORTRAN routine. The beam application and monitoring system (BAMS) ${ }^{6}$ of the accelerator, as well as the RW-3 slabs behind which the film specimens were positioned, were simulated by rectangular slabs of water with corresponding water-equivalent thicknesses. The total dose was scored using the USRBIN card with a detection area of $10 \times 10 \mathrm{~cm}^{2}$ with 200 bins in each $\mathrm{x}$ - and y-direction. In total, $3 \cdot 10^{8}$ particles were simulated, achieving a relative standard uncertainty below $2 \%$. A gamma index analysis with a $3 \mathrm{~mm} \mathrm{(3 \% )} \mathrm{criterion}$ was also performed to compare the film data with the simulated data.

\section{Results}

\subsection{Calibration curve}

The calibration function given by Eq. (3) was fitted to the data taken from the calibration fields of different doses for each color channel. The result is shown in Fig. 1. The fitting parameters and the normalized rootmean-square error (RMSE) ${ }^{7}$ of each fit are listed in Table 1.

The calibration curves fit the data points very well. When the calibration curves based on the carbon-irradiated film (Fig. 1, solid lines) are compared with the curves based on film irradiated using a LINAC (Fig. 1, dashed lines), both curves show a similar reaction, with the red curve having the steepest gradient and crossing first the green and then

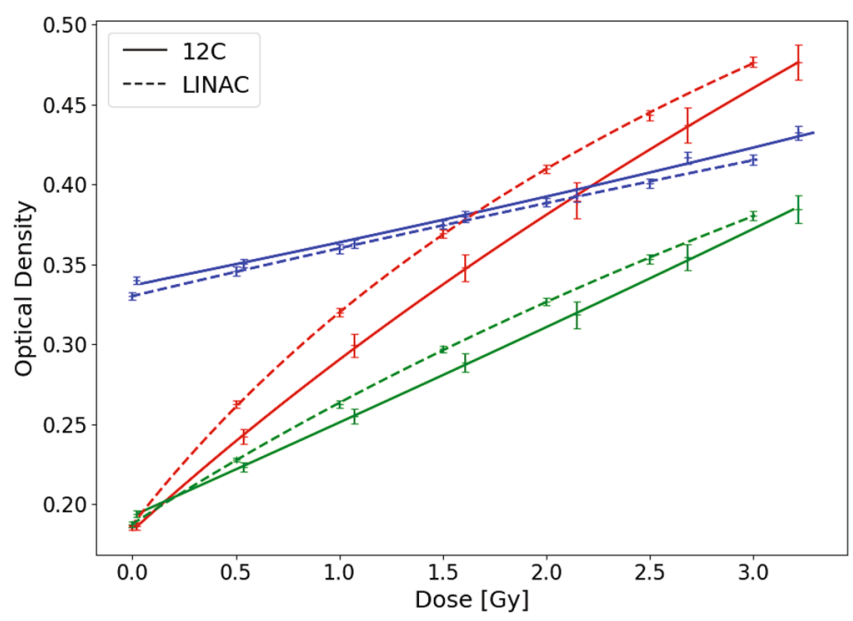

Fig. 1. Calibration curves for the red, green and blue color channels of carbonirradiated film specimens (solid lines) and for photon-irradiated film specimens (dashed lines). Error bars indicate the standard deviation of the pixel values from each other within the central ROI. (For interpretation of the references to color in this figure legend, the reader is referred to the web version of this article.)

\footnotetext{
5 MC: Monte Carlo

${ }^{6}$ BAMS: beam application and monitoring system

7 RMSE: root-mean-square error
} 
Table 1

Fitting parameters for Eq. (3) with one standard deviation errors and normalized RMSEs for ${ }^{12} \mathrm{C}$-irradiated film (initial beam energy $278.29 \mathrm{MeV} / \mathrm{u}$ ).

\begin{tabular}{lllll}
\hline Channel & $a$ & $b$ & $c$ & RMSE $\%$ \\
\hline Red & $2.14 \pm 0.03$ & $0.00106 \pm 0.00006$ & $3.19 \pm 0.05$ & 0.92 \\
Green & $6.10 \pm 0.15$ & $-0.028 \pm 0.018$ & $9.40 \pm 0.23$ & 0.76 \\
Blue & $9.3 \pm 0.3$ & $-0.196 \pm 0.018$ & $20.0 \pm 0.6$ & 3.49 \\
\hline
\end{tabular}

the blue curve. However, the signal for the LINAC-irradiated film specimens is higher for the red and green color channel, as shown by the corresponding calibration curves above the curves for ${ }^{12} \mathrm{C}$-irradiated specimens, and lower for the blue color channel. Furthermore, the curves' slope differs slightly between LINAC-irradiated and ${ }^{12} \mathrm{C}$-irradiated film, especially for the red color channel.

\subsection{Comparison of the signals of uncorrected and corrected film}

Figs. 2 and 3 show the original dose distribution from the red color channel and the corrected distributions of one of the irradiated film specimens. In total, we irradiated 14 film specimens at the SOBP region of the homogeneous irradiation field as described in Section 2.2, all of which showed a similar result when applying the triple channel analysis as presented in the following.

In Fig. 2, a number of artifacts can be observed in the original distribution (marked by the arrows). In addition, the signal in the plateau drops slightly in the upper left. For the corrected film specimen, the signal is more homogeneous and the artifacts have disappeared. The artifacts as well as the slight signal drop are clearly reflected in the distribution of the disturbance value $\delta$. In contrast, the higher signal on the left side ( $\mathrm{y} \approx 60 \mathrm{px}$ ), which is caused by additional material in the beam path $(0.5 \mathrm{~mm}$ PMMA, back of the $2 \mathrm{~cm}$ tall range modulator holder), is not affected by the correction. Calculating the ratio between the signal in this area and the signal in the middle of the field (between $\mathrm{x}, \mathrm{y}=85 \ldots 185 \mathrm{px}$ ) yields a value of 1.038 for the uncorrected distribution and 1.033 for the corrected distribution. The standard deviation for the inner $100 \times 100 \mathrm{px}\left(=35 \times 35 \mathrm{~mm}^{2}\right)$ was calculated for both distributions. For the uncorrected signal, this standard deviation was $1.9 \%$; for the corrected signal, it decreased to $1.3 \%$. The maximum deviation of the values in this area is $28.6 \%$ for the uncorrected film specimen and $9.8 \%$ for the corrected specimen.

The one-dimensional uncorrected signal shown in Fig. 3 drops at higher $\mathrm{x}$-values, as observed in the two-dimensional distribution. By comparison, the corrected signal is fairly constant within the plateau region of the field. For $\mathrm{x}=122$, a very sharp peak can be seen in the uncorrected signal; this peak vanishes in the corrected signal.

Comparing films that were irradiated under the same conditions (irradiation plan, position, measurement depth) a mean relative

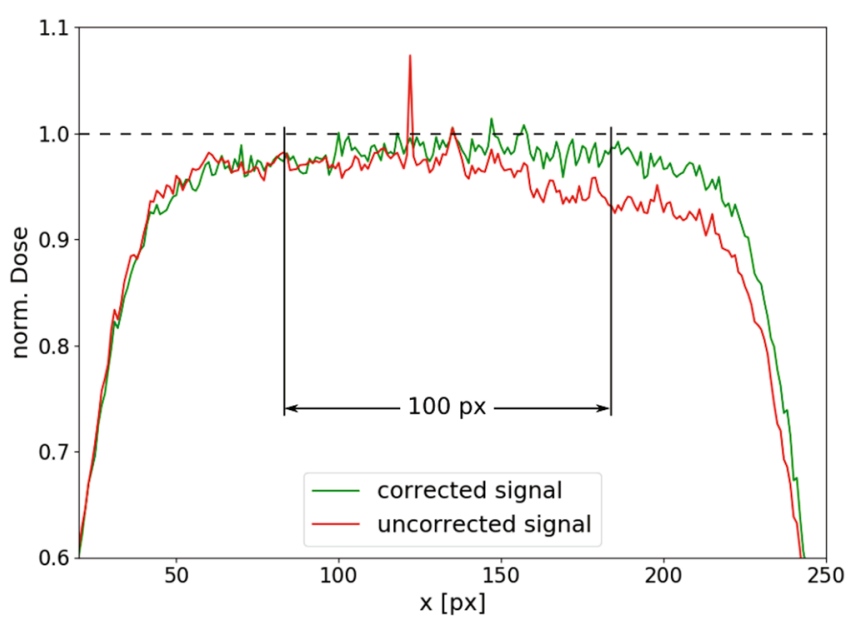

Fig. 3. Comparison of one-dimensional dose distributions taken from the center of an uncorrected film specimen (red) and the related corrected film signal (green). Coordinates are given as pixel numbers. (For interpretation of the references to color in this figure legend, the reader is referred to the web version of this article.)

standard deviation of $0.9 \%$ was found for the corrected dose distributions and $1.2 \%$ for the uncorrected dose distributions; the maximum relative standard deviations were $4.3 \%$ and $17.1 \%$ for the corrected and uncorrected distributions. The comparison between the uncorrected and the corrected film dose distributions is summarized in Table 2.

\subsection{Comparison of film data with IC array data}

Fig. 4 shows one-dimensional dose distributions across the $\mathrm{x}$-axes that were measured simultaneously with EBT3 and the IC array (as described in Section 2.6). The distributions were taken from the center of the field measured in the middle of the SOBP. Due to a very flat depth dose distribution here (as it is shown in [22]), the slight difference in the measurement depths of IC array and film can be neglected. To match the IC dimensions, the film signal was averaged over the inner seven rows of pixels, which correspond to a width of $2.5 \mathrm{~mm}$. For each curve, four measurements were performed and averaged. The standard deviation of these repeated measurements from each other was calculated as a measurement uncertainty; the mean relative standard deviation for the film measurements was $0.9 \%$, for the IC array data it was $0.3 \%$. Each data set was normalized to its central value; the pixel numbers were converted to $\mathrm{mm}$ to fit them to the coordinates of the IC array data.

Both distributions agree very well; differences of up to $0.4 \%$ lie within the measurement uncertainty of the film data. The IC array data has a standard deviation of the measured data points in the inner $35 \mathrm{x}$ (a)

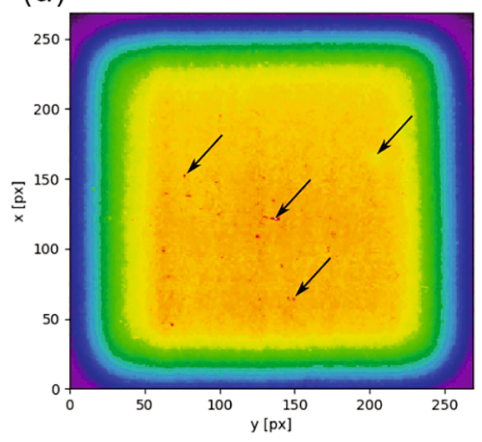

(b)

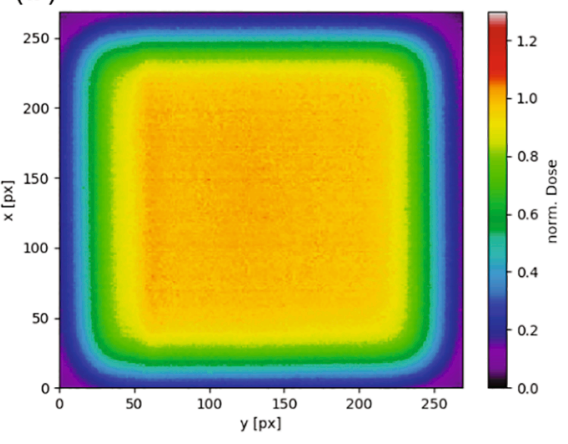

(c)

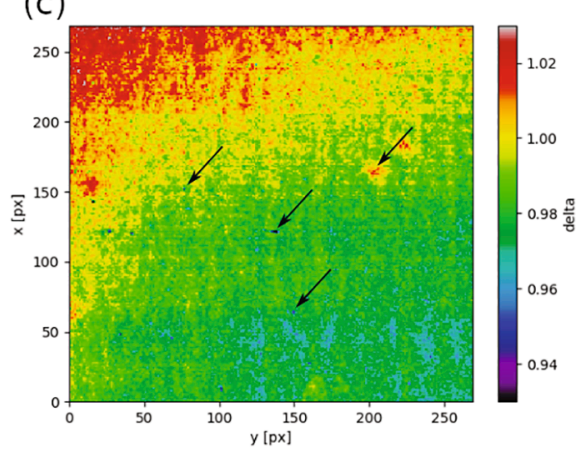

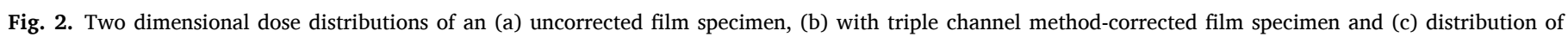

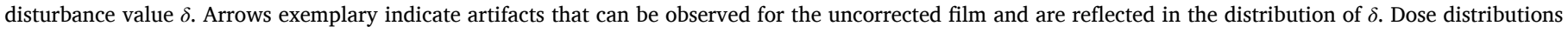
were normalized to the maximum; coordinates are given as pixel numbers. Measurements were taken in the middle of the SOBP. 


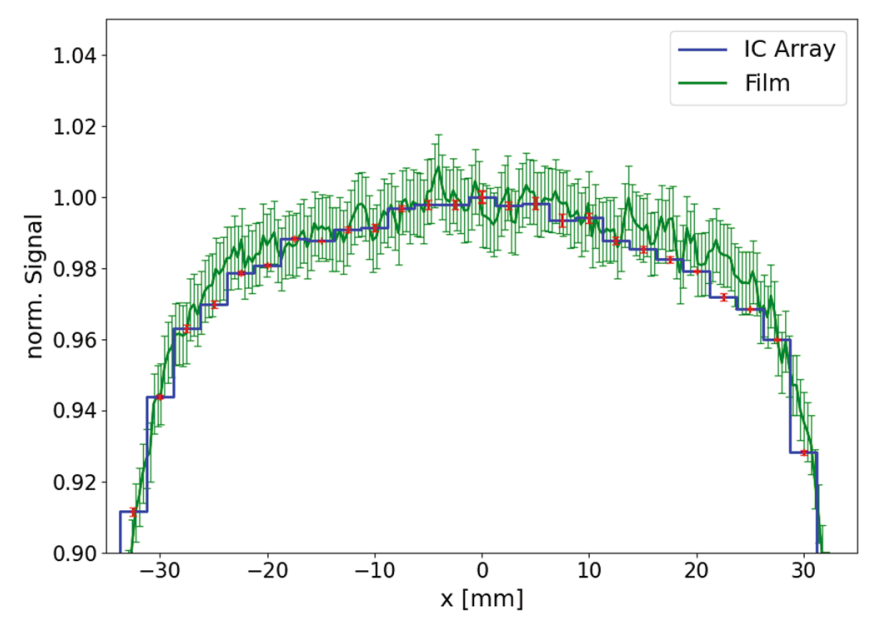

Fig. 4. Comparison of dose distributions measured with EBT3 and an IC array. Both were irradiated simultaneously. Error bars indicate the standard deviation of the repeated measurements from each other; based on these measurements, average value was obtained.

$35 \mathrm{~mm}^{2}$ of $0.8 \%$ and a maximum deviation of the points from each other of $3.1 \%$. For the film data, the standard deviation in the inner $100 \times 100$ px $\left(35 \times 35 \mathrm{~mm}^{2}\right)$ is $1.3 \%$; the maximum deviation is $9.8 \%$. The gamma index analysis yielded a pass rate of $85.4 \%$ when considering all measurement points and $97.6 \%$ when only taking points with dose values $>10 \%$ of the maximum dose into account.

The results for the comparison of dose distributions measured with EBT3 and the IC array are summarized in Table 2.

\subsection{Possible QA applications}

To investigate the applicability of EBT3 evaluated with the triple channel analysis for carbon-ion QA, further measurements were performed. Measurements with EBT3 film specimens positioned directly in front of the beam exit window of the nozzle were performed to investigate whether EBT3 could be used to check the mechanical integrity of the mini-ridge filter (ripple filter) in the beam line. A homogeneous irradiation field of $1.5 \mathrm{~Gy}$ and a field size of $150 \times 150 \mathrm{~mm}^{2}$ was applied. Film artifacts were removed by applying the triple channel analysis, thus improving the image quality and making the fluence inhomogeneities introduced by the ripple filter more visible. Fig. 5 shows the uncorrected dose distribution directly resulting from the optical density distribution for the red color channel as well as the corrected distribution after applying the triple channel analysis, measured with a film specimen positioned on the beam nozzle.

The ripple filter's pattern can be clearly observed in both the

Table 2

Summarized results for the comparison of uncorrected film dose distributions (directly taken from the red color channel signal), film dose distributions corrected by the triple channel analysis and dose distributions measured with the IC array. The standard deviation as well as the maximum deviation of dose values within the inner $100 \mathrm{x} 100 \mathrm{px}$ are given as a measure of field homogeneity. The standard deviation and maximum deviation between repeated measurements under the same irradiation conditions are stated below; the value for the IC array is taken from [22].

\begin{tabular}{llll}
\hline & uncorrected & corrected & IC array \\
\hline Field homogeneity: & & & \\
Std. dev. & $1.9 \%$ & $1.3 \%$ & $0.8 \%$ \\
Max. dev. & $28.6 \%$ & $9.8 \%$ & $3.1 \%$ \\
Repeated measurements: & & & \\
Std. dev. & $1.2 \%$ & $0.9 \%$ & $0.3 \%$ \\
Max. dev. & $17.1 \%$ & $4.3 \%$ & - \\
\hline
\end{tabular}

corrected and uncorrected film signals, but additional artifacts can be observed in the uncorrected signal. In addition, the uncorrected signal shows an asymmetric distribution with higher dose values towards the lower left, whereas the corrected signal is stable over nearly the entire field.

We tested the applicability of EBT3 for patient plan verification by irradiating one iso-energy slice with $212.12 \mathrm{MeV} / \mathrm{u}$ taken from the patient treatment plan (Section 2.2) and comparing the film signal to the signal measured with the IC array and simulated as described in Section 2.7. The result is shown in Fig. 6. The uncorrected film dose distribution was calculated from the film's netOD to take the film's zero dose signal into account. Each signal was normalized to its maximum.

It can be seen that both the uncorrected and the corrected film signals agree very well with the IC array signal as well as with the simulated data. Comparing the corrected film with the IC array signal and the simulated data, a pass rate for the gamma index analysis of $97.3 \%$ and $98.5 \%$ was derived. The pass rate for the comparison of the uncorrected film signal and the IC array (MC simulation) was at $94.6 \%(96.0 \%)$.

\section{Discussion}

\subsection{Calibration curve}

A similar reaction of EBT3 to different doses of photons and carbonion beams was observed, although the resulting calibration curves differ slightly. The decreased response of EBT to carbon ions compared to photons observed by Martisikova et al. [16] was also seen for the red and green color channel. Martisikova et al. found the response to carbon ions to be about $70 \%$ of the response to photons. Here, the $O D$ of the carbonion-irradiated film reached $90-96 \%$ of the photon-irradiated $O D$ for the red color channel and minimum $94 \%$ for the green color channel. For the blue color channel, a higher response was observed for the carbon-ionirradiated film, the signal was about $101 \%$ of the photon-irradiated $O D$.

The blue color channel showed the smallest differences between the film response to photon and carbon-ion irradiation. Using this color channel instead of the red color channel for carbon-ion film dosimetry could be advantageous due to the apparently smaller dependence of the film response on the irradiation modality. Further measurements using different initial beam energies of the carbon-ion beam would be of interest to further investigate this effect. Nevertheless, the calibration curve of the blue color channel shows the flattest course, which would mean reduced contrast in the resulting dose distribution when using this color channel to evaluate the measurement.

Furthermore, one must keep in mind that this does not eliminates the need to apply the triple-channel analysis, since the dose response in Fig. 1 was obtained by averaging the signal over a central ROI exactly to eliminate the influence of differences due to film non-homogeneity.

Due to the differences observed, calibration parameters for EBT3 measurements with carbon-ion beams should always be taken from ${ }^{12} \mathrm{C}$ irradiated film specimens.

\subsection{Corrected film signal}

Applying the triple channel analysis, a number of artifacts disappeared from the measured distribution (see arrows in Fig. 2 or peak for $x=122$ in Fig. 3). This artifact removal is also reflected in the lower maximum deviation of the inner measurement points from each other for the corrected signal (9.8\%) compared to the uncorrected signal (28.6\%). Similar point-like artifacts as well as their removal after applying the triple channel analysis were also observed for the other film specimen irradiated within this study. These artifacts were caused by contaminations in the film specimen such as dust particles or impurities within the film itself.

In addition to the point-like artifacts, the disturbance value $\delta$ showed an asymmetric distribution dropping from the upper left to the lower right in Fig. 2 and from low to high $\mathrm{x}$-values in Fig. 3. As the irradiated 

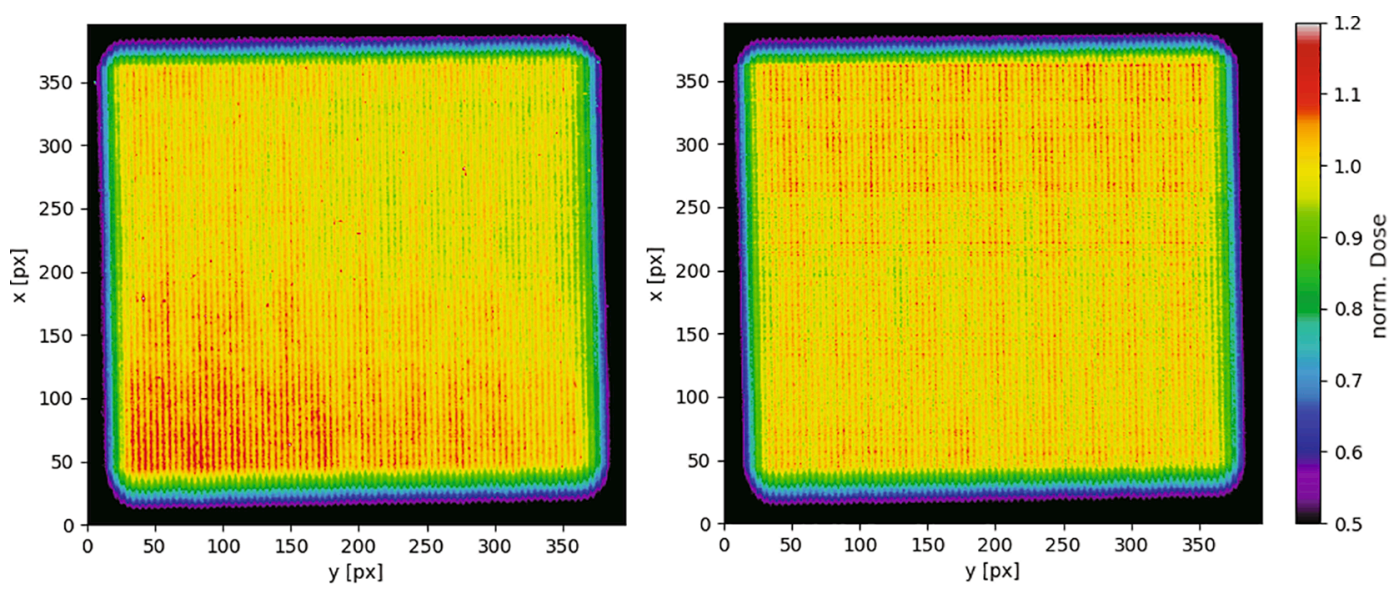

Fig. 5. Uncorrected (left) and corrected (right) film dose distributions of an EBT3 positioned directly on the beam nozzle.

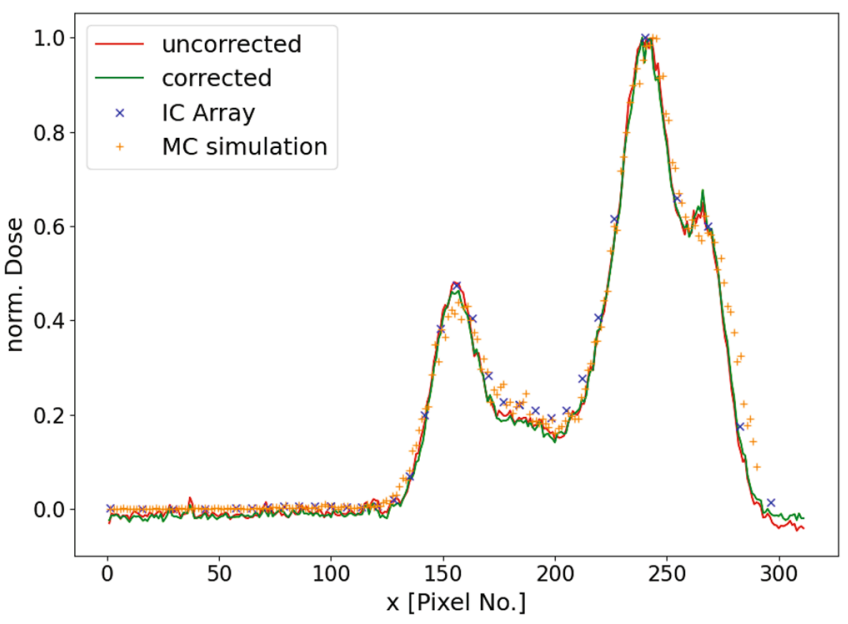

Fig. 6. Corrected and uncorrected 1D film dose distribution across the x-axis of the irradiated patient treatment plan and comparison with the distribution measured with the IC array and simulated data. Measurement took place at a $75 \mathrm{~mm}$ water-equivalent depth (realized by RW-3 slabs); each data set was normalized to its maximum.

dose distribution shows a stable course over its plateau area, this drop is due to an asymmetric response of the film measurement, which could be caused by a variation in the film thickness or an asymmetry in the read out system. As such a drop was observed for all film specimen irradiated within this study, the asymmetry in the read out system is the most probable explanation. This asymmetry is compensated by the triple channel analysis, as the drop is not observable in the corrected film signal.

In contrast, the higher signal at the left caused by additional material in the beam path is not affected by the correction. This is also shown by the calculated ratios of the higher signal and the signal at the middle of the field being in the same order of magnitude for both the uncorrected and corrected signals. This shows that the applied triple channel analysis only corrects for artifacts of the film itself, leaving out all inhomogeneities of the actual dose distribution.

The comparison of repeated film measurements under the same irradiation conditions showed a decreased standard deviation of $0.9 \%$ between the measured dose distributions after applying the triple channel analysis. In contrast, the mean standard deviation of $1.2 \%$ between the uncorrected dose distributions was influenced by film artifacts causing differences between the film measurements; this is especially reflected in the high maximum standard deviation of $17.1 \%$. For the same irradiation field, a mean standard deviation of $0.26 \%$ between repeated measurements using the IC array was presented in [22]. The discrepancy to the $0.9 \%$ found here can be explained by the noisier film signal compared to the IC array as it was presented in Section 3.3. These results prove the triple channel analysis to correct for non-dose-related artifacts allowing a better comparison between repeated measurements.

\subsubsection{Analysis of measurement uncertainty}

Six films were irradiated in the same conditions and the $O D$ over a region of interest of $100 \times 100 \mathrm{px}\left(3.5 \times 3.5 \mathrm{~cm}^{2}\right)$ was calculated, resulting in $2.4 \%$ for the uncorrected film and $2.3 \%$ for the corrected film. This uncertainty is not significantly reduced by the triple channel algorithm because the value is based on the average over a large area. Therefore, this represents the uncertainty associated with the film and irradiation reproducibility only.

Taking the uncertainty of the dose calibration and the correlation of the fitting parameters (determined from the fit's covariance matrix) into account, an uncertainty budget was determined according to the Guide to the Expression of Uncertainty in Measurement (GUM) [37]. All contributions as well as the resulting overall uncertainty of the resulting film dose values for the red color channel are given in Table 3.

This uncertainty budget does not take uncertainties due to film artifacts or the effect of the triple channel analysis into account, as these do not affect the whole dose distribution. As a measure of possible uncertainties caused by artifacts, we compared the uncorrected and corrected dose distributions of each film specimen irradiated with the homogeneous irradiation plan. Maximum differences between the pixel values of the corrected and the uncorrected film signal were found to be between $10 \%$ and $38 \%$. This means, without correction, artifacts can cause a deviation in the measured dose by up to $38 \%$.

\section{Table 3}

Combined standard measurement uncertainty budget for the determination of absorbed dose in EBT3 film measurements from the red color channel (uncorrected film signal). For the film signal $O D$, the relative standard deviation of the data for a $100 \times 100$ px ROI of six identically exposed films is given. Using the triple channel analysis, the uncertainty in the film signal $O D$ is reduced only to $2.3 \%$ because artifacts do not influence much the average over the large ROI of 100 x 100 px.

\begin{tabular}{ll}
\hline Quantity & Rel. standard uncertainty/\% \\
\hline Film signal $O D$ & 2.4 \\
Calibration & \\
Fitting parameter $a$ & 1.6 \\
Fitting parameter $b$ & 5.5 \\
Fitting parameter $c$ & 1.7 \\
Dose $D$ & 6.7 \\
\hline
\end{tabular}




\subsection{Comparison of film data with IC array data}

The distributions measured simultaneously with the IC array and the film showed good qualitative agreement; the differences observed were within the measurement uncertainty. This agreement was also quantitatively reflected by a high gamma pass rate of $97.6 \%$ within the high dose region (dose values $>10 \%$ of the maximum dose). Due to volume averaging, the IC signal had a smaller contribution of high spatial frequencies. This was reflected by the noisier film signal compared to the IC array signal and the higher standard deviation of the film measurement points within the center of the field from each other $(1.3 \%$, IC array: $0.8 \%)$.

\subsection{Possible $Q A$ applications}

As a possible application of EBT3 film corrected with the triple channel analysis method, we investigated the visibility of ripple filter inhomogeneities on film specimens that were positioned directly in front of the beam exit window of the nozzle. For both the corrected and uncorrected signal, the ripple filter's pattern was clearly visible. However, due to artifacts in the signal caused by impurities on and within the film, it was not possible to distinguish possible defects in the ripple filter and film artifacts for the uncorrected film signal. This is possible only for the corrected film signal. As the applied irradiation field results in a homogeneous dose distribution, the asymmetry in the uncorrected film signal can be explained by a variation in the film thickness, resulting in a position-dependent response. This is compensated by the triple channel analysis.

This measurement demonstrates how EBT3 can be used to check the ripple filter only when applying the triple channel analysis method to the film signal.

The comparison of the film signal with the IC array data as well as the MC simulated data for the patient treatment plan shows that EBT3 can be used to verify single iso-energy slices taken from treatment plans if corrected by applying the triple channel analysis method. The good agreement between the corrected film signal and the IC array signal as well as the simulated data is also indicated by the high gamma pass rate of $97.3 \%$ and $98.5 \%$, this rate is slightly reduced for the comparison with the uncorrected film signal ( $94.6 \%$ and $96.0 \%)$.

\section{Conclusion}

The presented study demonstrated the need for the triple channel analysis to eliminate film artifacts in carbon-ion film dosimetry. The use of the analysis significantly improved the image quality of lateral dose distributions, as demonstrated by a reduction in the relative standard deviation of measured doses within a homogeneous irradiation field from $1.9 \%$ to $1.3 \%$, and a reduction in the maximum deviation from $28.6 \%$ to $9.8 \%$ (see Table 2). This improvement is necessary if fine structures associated with the carbon-ion beams are to be captured by the film measurements. The corrected film doses were also shown to agree with IC array data within $0.4 \%$ in the low gradient region (central $40 \times 40 \mathrm{~mm}^{2}$ region). The applicability of EBT3 evaluated with the triple channel analysis was also demonstrated for QA measurements in carbon-ion beams for cases of uniform radiation quality, such as an inspection of ripple filters and a verification of patient plans for single isoenergy slices.

This study highlights the needs for the triple channel analysis to eliminate artifacts associated with film inhomogeneity, if the fine structures observed in carbon-ion beams are to be properly captured by the radiochromic films.

\section{Acknowledgements}

We would like to thank Uli Weber from GSI for providing us with the irradiation plan for the calibration fields and Stephan Brons and his colleagues from HIT for their help with technical issues concerning the irradiations.

\section{Appendix A. Supplementary data}

Supplementary data associated with this article can be found, in the online version, at https://doi.org/10.1016/j.ejmp.2021.06.009.

\section{References}

[1] Stevens MA, Turner JR, Hugtenburg RP, Butler PH. High-resolution dosimetry using radiochromic film and a document scanner. Phys Med Biol 1996;41:2357.

[2] Butson MJ, Yu PKN, Cheung T, Metcalf P. Radiochromic film for medical radiation dosimetry. Mater Sci Eng 2003;41:61.

[3] Podgorsak E. Review of Radiation Oncology Physics: A Handbook for Teachers and Students. International Atomic Energy Agency 2003.

[4] van Battum LJ, Hoffmans D, Piersma H, Heukelom S. Accurate dosimetry with Gafchromic ${ }^{\mathrm{TM}}$ EBT film of a photon beam in water: What level is achievable? Med Phys 2008;35(2):704-16.

[5] Hartmann Bernadette, Martiš́́ková Mária, Jäkel Oliver. Technical note: homogeneity of Gafchromic TM EBT2 film. Med Phys 2010;37(4):1753-6.

[6] Micke A, Lewis D, Yu X. Multichannel film dosimetry with nonuniformity correction. Med Phys 2011;38(5):2523.

[7] Mayer R, Ma F, Miller R, Belard A, McDonough J, O'Connell J. Enhanced dosimetry procedures and assessment for EBT2 radiochromic film. Med Phys 2012;39(4): 2147.

[8] Mendez I, Peterlin P, Hudej R, Strojnik A, Casar B. On multichannel film dosimetry with channel-independent perturbations. Med Phys 2014;41:011705.

[9] Mendez I. Model selection for radiochromic film dosimetry. Phys Med Biol 2015; 60:4089.

[10] Hayashi N, Watanabe Y, Malmin R, Kato H. Evaluation of triple channel correction acquisition method for radiochromic film dosimetry. J Radiat Res 2012;53(6):930.

[11] van Hoof SJ, Granton PV, Landry G, Podesta1 M, Verhaegen F. Evaluation of a novel triple-channel radiochromic film analysis procedure using EBT2. Phys Med Biol 2012;57:4353.

[12] Li Y, Chen L, Zhu J, Liu X. The combination of the error correction methods of Gafchromic ${ }^{\text {TM }}$ EBT3 film. Plos One 2017;12(7).

[13] Palmer A, Bradley D, Nisbet A. Evaluation and implementation of triple-channel radiochromic film dosimetry in brachytherapy. J Appl Clin Med Phys 2014;15(4): 280.

[14] Haberer T, Becher W, Schardt D, Kraft G. Magnetic scanning system for heavy ion therapy. Nucl Instrum Methods Phys Res A 1993;330:296.

[15] Castriconi R, Ciocca M, Mirandola A, Sini C, Broggi S, Schwarz M, Fracchiolla F, Martišíková M, Aricó G, Mettivier1 G, Russo P. Dose-response of EBT3 radiochromic films to proton and carbon ion clinical beams. Phys Med Biol 2017; 62:377.

[16] Martišíková M, Jäkel O. Dosimetric properties of Gafchromic TM EBT films in monoenergetic medical ion beams. Phys Med Biol 2010;55:3741.

[17] Martišíková M, Jäkel O. Dosimetric properties of Gafchromic TM EBT films in medical carbon ion beams. Phys Med Biol 2010;55:5557.

[18] Reinhardt S, Hillbrand M, Wilkens J, Assmann W. Comparison of Gafchromic EBT2 and EBT3 films for clinical photon and proton beams. Med Phys 2012;39(8).

[19] Goma C, Andreo P, Sempau J. Spencer-Attix water/medium stopping-powerratios for the dosimetry of proton pencil beams. Phys Med Biol 2013;58:2509.

[20] Gafchromic. Gafchromic EBT3 dosimetry film specifications; 2019. http://www. gafchromic.com/ (accessed 03/29/19).

[21] Haberer T, Debus J, Eickoff H, Jäkel O, Schulz-Ertner D, Weber U. The Heidelberg ion therapy center. Radiother Oncol 2004;73(2):S186.

[22] Holm KM, Weber U, Simeonov Y, Krauss A, Jäkel O, Greilich S. 2D range modulator for high-precision water calorimetry in scanned carbon-ion beams. Phys Med Biol 2020;65:215003.

[23] Simeonov Y, Weber U, Penchev P, Ringbæk T, Schuy C, Brons S, EngenhartCabillic R, Bliedtner J, Zink K. 3D range-modulator for scanned particle therapy: development, Monte Carlo simulations and experimental evaluation. Phys Med Biol 2017;62:7075.

[24] Niroomand-Rad A, Blackwell CR, Coursey BM, Gall KP, McLaughlin WL, Galvin JM, et al., Radiochromic film dosimetry: Recommendations of AAPM Radiation Therapy Committee Task Group 55. Med Phys 1998;25(11):2093.

[25] Fuss M, Sturtewagen E, De Wagter C, Georg D. Dosimetric characterization of Gafchromicc EBT film and its implication on film dosimetry quality assurance. Phys Med Biol 2007;52:4211.

[26] Devic S, Seuntjens J, Sham E, Podgorsak EB, Schmidtlein CR, Kirov AS, Soares GC. Precise radiochromic film dosimetry using a flat-bed document scanner. Med Phys 2005;32(7):2245.

[27] Lewis D, Micke A, Yu X. An efficient protocol for radiochromic film dosimetry combining calibration and measurement in a single scan. Med Phys 2012;39(10): 377.

[28] Palmer A, Bradley D, Nisbet A. Evaluation and mitigation of potential errors in radiochromic film dosimetry due to film curvature at scanning. J Appl Clin Med Phys 2015;16(2):425.

[29] Gafchromic. Efficient protocols for accurate radiochromic film calibration and dosimetry; 2019. http://www.gafchromic.com/ (accessed 03/29/19). 
[30] Alexander K. Development of a novel readout system for radiochromic film dosimetry. Master's thesis; 2014.

[31] Devic S, Aldelaijan S, Mohammed H, Tomic N, Liang L-H, DeBlois F, Seuntjens J. Absorption spectra time evolution of EBT-2 model Gafchromic film. Med Phys 2010;37(5):2207.

[32] Doll L. Characterization of a high-resolution ionization chamber array in clinical carbon ion and proton beams. Master's thesis. Germany: Department of Physics, University of Heidelberg; 2017.

[33] PTW Freiburg. Octavius 1000 SRS, 2019. http://www.ptw.de/2287.html (accessed 04/04/19).
[34] Bauer D. Study of the Octavius ionization chamber array as a film replacement for clinical ion beam quality assurance. Master's thesis. Germany: Department of Physics, University of Heidelberg; 2018.

[35] Low Daniel A, Harms William B, Mutic Sasa, Purdy James A. A technique for the quantitative evaluation of dose distributions. Med Phys 1998;25(5):656-61.

[36] Ferrari A, Sala PR, Fasso A, Ranft J. Fluka: a multi-particle transport code (Program version 2014).

[37] Joint Committee for Guides in Metrology. Evaluation of measurement data - Guide to the expression of uncertainty in measurement. JCGM 100:2008; 2008. 